\title{
The Impact of Quick Charging Stations on the Route Planning of Electric Vehicles
}

\author{
Bülent Çatay*, Merve Keskin \\ Faculty of Engineering and Natural Sciences, Sabanci University \\ Tuzla 34956, Istanbul, Turkey \\ \{ catay, mervekeskin \}@sabanciuniv.edu \\ * Corresponding author
}

\begin{abstract}
Many companies have a growing interest in utilizing alternative fuel vehicles in their logistics operations due to increasing environmental concerns in developed countries. Consequently, green vehicle routing problems have attracted more attention in the literature. The Electric Vehicle Routing Problem (EVRP) is one such problem where the customers are served using an electric vehicle (EV) fleet. In this problem, the energy on the battery of the $\mathrm{EV}$ is consumed proportionally with distance traveled and the EV may need recharging en route in order to complete its tour. In this study, we consider a variant of EVRP where the customers are associated with service time windows and the stations may be equipped with normal and quick charging systems. In the quick charge case, the battery is recharged with the same energy in a shorter time but at a higher cost. Our objective is to minimize energy costs while operating minimum number of vehicles. We formulate the mathematical programming models of the single and multiple charger cases and solve them using a commercial solver. Our aim is to investigate the complexity of the problems and analyze the potential benefits associated with the quick charging option.
\end{abstract}

Keywords-electric vehicle; vehicle routing problem; quick charge; alternative fuel vehicles, green logistics

\section{INTRODUCTION}

Transportation systems account for about $20-25 \%$ of global energy consumption and $\mathrm{CO}_{2}$ emissions. The major contributor is the road transportation with a share of $75 \%$ [1]. Fossil fuels, mainly gasoline and diesel, correspond to $95 \%$ of the energy sources used in global transport operations. In 2013, about $27 \%$ of total greenhouse gas (GHG) emissions in the US were transport related [2]. 74\% of the domestic freight in 2012 was moved by trucks and the freight volume is expected to grow by $39 \%$ in 2040 [3]. On the other hand, transportation accounts for $63 \%$ of fuel consumption and $29 \%$ of all $\mathrm{CO}_{2}$ emissions in the EU. The expected growth of freight transport in 2050 is around $80 \%$ compared to 2005 [4]. These figures reveal that transportation will continue to be a major and still growing source of GHGs. Hence, governments have started setting new targets and implementing new environmental measures for reducing emissions and fuel resource consumptions. Since transportation plays a major part in GHG emissions, the regulations encourage the use of alternative fuel vehicles such as solar, electric, biodiesel, LNG, CNG vehicles. EU countries such as Netherlands, Norway, and
Germany have adopted new motions that will ban sales of fossil fuel cars in the next 10-15 years [5,6]. City logistics in major European urban centers will be $\mathrm{CO}_{2}$-free by 2030 [1]. All these developments have increased the interest in EV technology and applications, and as a result, route optimization for EV fleets has become a challenging and popular problem in the Vehicle Routing Problem (VRP) literature due to the additional complexities it brings.

EVs can be classified as battery electric vehicles (BEV), hybrid electric vehicles (HEV), and fuel-cell electric vehicles (FCEV). In this study, we refer to an EV as a commercial road $\mathrm{BEV}$. The main advantages of EVs are zero tailpipe emission, high efficiency, low operating noise, less maintenance requirements, and gaining some of the energy through regenerative breaking whereas the major drawbacks are limited driving range, the limited availability of recharging stations, and long battery recharging times.

There are different ways for recharging an EV, including conductive charging, inductive charging, and battery swapping. The most common method is conductive charging using a cable and vehicle connector. In the inductive charging, the power is transferred to the battery magnetically via an on-board charger without needing any cables or connectors [7]. Battery swapping refers to replacing the empty battery with a fully charged one in swap station. Catenary wires is another recharging method where the energy can be transferred using a pantograph device which slides along the electric wires. Its most common application is on public electric buses.

The battery recharging times are dependent on the battery type, charging equipment and charging level. Charging levels can be classified into three categories: level $1(1.4 \mathrm{~kW}$ to $1.9 \mathrm{~kW})$, level 2 (4 kW to $19.2 \mathrm{~kW}$ ), and level $3(50 \mathrm{~kW}$ to $100 \mathrm{~kW}$ ) [7]. The last is also called as quick charging. The charge durations are linear with respect to time at the first phase of charging which corresponds to almost full battery while the second phase is nonlinear and can take hours to obtain a fully charged battery [8].

HEVs can be classified as parallel, series, series-parallel, and complex according to their powertrain architecture [9]. A plug-in hybrid electric vehicle (PHEV) is an HEV which is equipped with 
a rechargeable battery and can run using both electric motor and internal combustion engine (ICE). In series type vehicles, the ICE is used to power a generator and the propulsion is obtained from the electric motor whereas in the parallel type, both the ICE and the electric motor are used in the propulsion. The main advantage of PHEVs is their ability to move using fuel when the battery is depleted [9].

FCEVs use hydrogen as input and the electricity is produced by a fuel cell via a chemical reaction. The generated electricity is used to charge a battery or power the electric motor. The output of this reaction is simply water [9]. The US Department of Energy reported that fuel cells can convert approximately 50\% of hydrogen's energy to electricity and have a durability of 10,000 operating hours [10]. These constitute the main drawbacks of FCEVs.

In this study, we address the Electric VRP with Time Windows (EVRPTW) using a homogeneous fleet of EVs and present 0-1 mixed integer linear programming models by considering both single (slow) and multiple (quick) chargers. In the latter, we assume that the stations are equipped with multiple chargers which vary in power supply, power voltage, and maximum current options, which affect the recharge duration and the cost of energy. To the best of our knowledge, this is the first study that extends the modeling of EVRPTW to include multiple charging options. We formulate the mathematical models for both single and multiple chargers, and solve the small benchmark instances from the literature. Our aim is to investigate the advantages of quick charging option and compare the solutions against those achieved with the single charger solutions.

The remainder of the paper is organized as follows: Section 2 provides a brief review of the related literature. Section 3 describes the problem and presents mathematical programming formulations. Section 4 designs the computational study and discusses the results of the experiments. Finally, Section 5 provides the concluding remarks and future research directions.

\section{RELATED LITERATURE}

Recharging VRP (RVRP) was introduced as a new variant of VRP where the EVs can be recharged at selected customer locations while servicing the customer [11]. The charging time was assumed constant and the battery was either fully or partially (80\%) charged. Wang and Cheu (2012) investigated the operations of an electric taxi fleet [12]. The charging times were constant and the battery was full after recharging. An AFV routing problem with time-windows was studied by Omidvar and Tavakkoli-Moghaddam (2012) where the refueling times were assumed constant [13]. Worley and Klabjan (2012) addressed the problem of locating recharging stations and designing EV routes simultaneously [14].

Erdogan and Miller-Hooks (2012) considered the routing of AFVs where the objective was to minimize total distance travelled [15]. They referred to this problem as Green VRP (GVRP). In GVRP, EVs had unlimited cargo capacity, refueling times were assumed fixed and after refueling the tank became full.
Schneider at al. (2014) extended this problem within the context of EVRPTW, provided a new mathematical formulation and developed a hybrid metaheuristic solution procedure that combined the Variable Neighborhood Search (VNS) with tabu search [16]. Desaulniers et al. (2016) tackled the same problem by considering four recharging strategies (single-full recharge, single-partial recharge, multiple-full recharge, and multiplepartial recharge) and developed a branch-price-and-cut algorithm to solve it to optimality [17].

Keskin and Çatay (2016) developed an Adaptive Large Neighborhood Search (ALNS) method to solve EVRPTW by allowing partial recharges [18]. In the partial recharge case, the battery is recharged at any state of charge (SoC) and its duration is proportional to the energy transferred. Partial recharging was also addressed in Felipe et al. (2014) where different charging technologies were available [19]. The problem did not involve time windows but EVs had capacity and routes have duration limits. EVRPTW was also studied by considering the minimization of total travel, waiting and recharging time with minimum number of vehicles [20]. A VNS Branching approach was proposed to solve the problem.

Recently, EVRPTW was extended to the routing of a mixed fleet of EVs and ICE vehicles, which minimizes the energy consumption dependent on speed, gradient, and cargo load distribution [21]. Fleet size and mix vehicle routing problem with time windows was also addressed where the fleet consisted of EVs with different capacities [22]. In both studies ALNS was utilized to solve the problem.

We refer the interested reader to [23,24] for a comprehensive overview of goods distribution with EVs.

\section{PROBLEM DESCRIPTION AND MODELS}

\section{A. Problem Definition}

EVRPTW with partial recharges involves a set of customers with known demands, delivery time windows, and service durations, and a set of recharging stations. The deliveries are performed by a homogeneous fleet of EVs with fixed loading capacity and limited driving range. While the vehicle is traveling, the battery energy is consumed proportionally with the distance traversed. So, the EV may need to recharge its battery en route in order to complete its tour. The battery is recharged at any SoC, the duration depends on the charger type and is proportional to the amount of energy transferred.

Fig. 1 illustrates an example involving eight customers (C1C8), four stations (S1-S4), and the depot (D). The stations are equipped with three different chargers. The battery icons show the battery SoC when the vehicle arrives at a customer or a station and when it departs from the station after having its battery recharged. The charge connector icon placed next to the stations indicates the type of charging performed where L1, L2, and L3 refer to Level 1, Level 2, and Level 3, respectively. The EV traveling to the West visits first $\mathrm{C} 8$, then has its battery recharged at $\mathrm{S} 4$ using Level 2 charging before visiting $\mathrm{C} 7$ and $\mathrm{C} 6$. The EV 


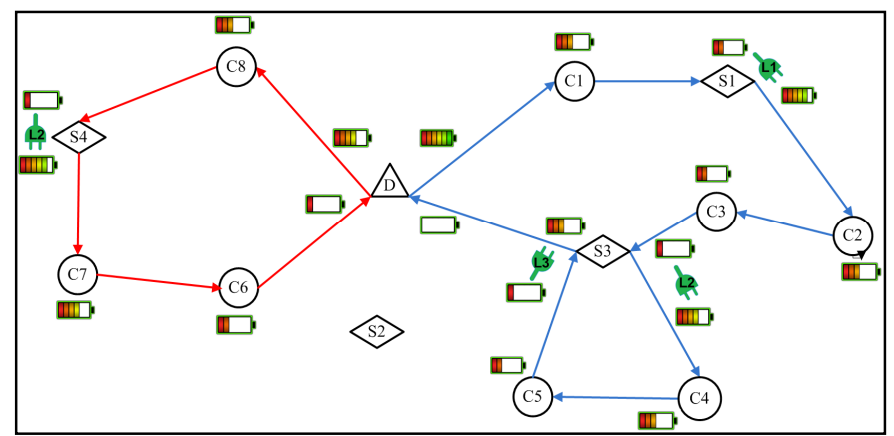

Fig. 1: An illustrative example of routes with quick charging stations

traveling to the East has its battery recharged three times along the tour. The first is a Level 1 charging at S1, the next two are at S3 with Level 2 charging first and then Level 3 charging next. It is important to note that a station can be visited multiple times by the same (e.g. S3) or different EVs and not all stations are necessarily visited (e.g. S2).

\section{B. Mathematical Models}

We provide two different mathematical models related to two different problems. The first is a $0-1$ integer programming model presented in [15] for the partial recharge case using only a single charger type at the stations. The second extends the problem by allowing multiple charger types. Let $V=\{1, \ldots, N\}$ denote the set of customers and $F$ denote the set of recharging stations. Since a recharging station may be visited more than once, we create copies of each station. So, $F^{\prime}$ is the set of vertices generated to permit several visits to each vertex in the set $F$. Vertices 0 and $N+1$ denote the depot and every route starts at 0 and ends at $N+$ 1 . Let $V^{\prime}$ be a set of vertices with $V^{\prime}=V \cup F^{\prime}$. In addition, we define $F_{0}^{\prime}=F^{\prime} \cup\{0\}, V_{0}^{\prime}=V^{\prime} \cup\{0\}$ and $V_{N+1}^{\prime}=V^{\prime} \cup\{N+1\}$. Now we can define the problem on a complete directed graph $G=$ $\left(V_{0, N+1}^{\prime}, A\right)$ with the set of $\operatorname{arcs} A=\left\{(i, j) \mid i, j \in V_{0, N+1}^{\prime}, i \neq j\right\}$ where $V_{0, N+1}^{\prime}=\{0\} \cup V_{N+1}^{\prime}$. Each arc is associated with a distance $d_{i j}$ and travel time $t_{i j}$. The battery charge is consumed at a rate of $h$ and every traveled arc consumes $h \times d_{i j}$ of the remaining battery. Each vertex $i \in V$ has positive demand $q_{i}$, service time $s_{i}$, and time window $\left[e_{i}, l_{i}\right]$. Each EV is associated with cargo capacity $C$ and battery capacity $Q$. At a recharging station, the battery is charged at a recharging rate of $g$. The decision variables, $\tau_{i}, u_{i}$, and $y_{i}$ keep track of the arrival time, remaining cargo level and remaining charge level at vertex $i \in V_{0, N+1}^{\prime}$, respectively. The binary decision variable $x_{i j}$ takes value 1 if arc $(i, j)$ is traversed and 0 otherwise. Model 1 which assumes single charger type is formulated as follows:

Model 1:

$\min \sum_{i \in V_{0}^{\prime}, j \in V_{n+1}^{\prime}, i \neq j} d_{i j} x_{i j}$ subject to

$$
\begin{array}{lc}
\sum_{j \in V_{n+1}^{\prime}, i \neq j} x_{i j}=1 & \forall i \in V \\
\sum_{j \in V_{n+1}^{\prime}, i \neq j} x_{i j} \leq 1 & \forall i \in F^{\prime} \\
\sum_{i \in V_{0}^{\prime}, i \neq j} x_{i j}-\sum_{i \in V_{n+1}^{\prime}, i \neq j} x_{j i}=0 & \forall j \in V^{\prime} \\
\tau_{i}+\left(t_{i j}+s_{i}\right) x_{i j}-l_{0}\left(1-x_{i j}\right) \leq \tau_{j} & \forall i \in V_{0}, \forall j \in V_{n+1}^{\prime}, \quad i \neq j \\
\tau_{i}+t_{i j} x_{i j}+g\left(Y_{i}-y_{i}\right)-\left(l_{0}+g Q\right)\left(1-x_{i j}\right) \leq \tau_{j} \\
e_{j} \leq \tau_{j} \leq l_{j} \quad \forall i \in F^{\prime}, \forall j \in V_{n+1}^{\prime}, i \neq j \\
0 \leq u_{j} \leq u_{i}-q_{i} x_{i j}+C\left(1-x_{i j}\right) \\
0 \leq u_{0} \leq C \quad \forall i \in V_{0}^{\prime}, \forall j \in V_{n+1}^{\prime}, i \neq j \\
0 \leq y_{j} \leq y_{i}-\left(h \cdot d_{i j}\right) x_{i j}+Q\left(1-x_{i j}\right) \\
\forall i \in V, \forall j \in V_{n+1}^{\prime}, i \neq j \\
0 \leq y_{j} \leq Y_{i}-\left(h \cdot d_{i j}\right) x_{i j}+Q\left(1-x_{i j}\right) \\
\forall i \in F_{0}^{\prime}, \forall j \in V_{n+1}^{\prime}, i \neq j \\
y_{i} \leq Y_{i} \leq Q \quad \forall i \in F_{0}^{\prime} \\
x_{i j}=0 \quad \forall i, j \in F^{\prime}, i \neq j \\
x_{i j} \in\{0,1\} \quad \forall i \in V_{0}^{\prime}, \forall j \in V_{n+1}^{\prime}, i \neq j
\end{array}
$$

The objective function (1) minimizes total distance traveled. Constraints (2) and (3) handle the connectivity of customers and visits to recharging stations, respectively. The flow conservation constraints (4) enforce that the number of outgoing arcs equals to the number of incoming arcs at each vertex. Constraints (5) and (6) ensure the time feasibility of arcs leaving the customers (and the depot), and the stations, respectively. Constraints (7) enforce the time windows of the customers and the depot. In addition, constraints (5)-(7) eliminate the sub-tours. Constraints (8) and (9) guarantee that demand of all customers are satisfied. Constraints (10) and (11) keep track of the battery state of charge and make sure that it is never negative. Constraints (12) determine the battery state of charge after the recharge at a station and make sure that the battery state of charge does not exceed its capacity. Constraints (13) prevent two consecutive recharges, i.e. after departing from a station the EV goes either to a customer or back to depot. This is a practical assumption when considering last mile deliveries in urban logistics. Finally, constraints (14) define the binary decision variables.

In the single charge model above, the depot is represented by one vertex. When the stations are equipped with different charging technologies we need to monitor the quantity of energy that each EV is recharged and the type of charger utilized because the costs differ. We manage this in the mathematical model by 
defining dummy sets of departure depot and arrival depot vertices, $D D$ and $A D$, respectively. The size of $D D$ and $A D$ is equal to the number of EVs (routes). These vertices are created artificially to keep track of battery SoC of the EV when it returns to the depot. In reality, there is a single physical depot where all EVs depart from and arrive at. We assume that each station is equipped with the same charger types but the EV is recharged by using only one of the chargers at each visit to the station. Note that this assumption can be easily relaxed but may not be practical in the real environment.

To allow charging at different speeds, we create a dummy station for each charger type. For instance, if the stations are equipped with two different chargers, normal and quick, we represent each of these chargers as a separate station at the same location. Basically, we create copies of stations to allow both multiple visits of the EVs and multiple charging options. So, the set $F^{\prime}$ in the EVRPTW with quick charging model represents all these stations. Note that this may increase the number of variables significantly and make the problem a lot harder to solve.

Let $c^{i}$ denote the cost of unit energy charged at station $i$. We define $c^{0}$ as the unit energy cost associated with the slowest (cheapest) charger type. We assume that the depot is equipped with this charger type since the EVs can be recharged fully overnight without needing quick charging. Then, the quick charging case can be formulated as follows:

$$
\begin{aligned}
& \text { Model 2: } \\
& \begin{array}{r}
\min \sum_{i \in F^{\prime}} c^{i}\left(Y_{i}-y_{i}\right) \\
\quad+c^{0}\left(Q \sum_{i \in D D} \sum_{j \in V^{\prime}} x_{i j}-\sum_{i \in A D} y_{i}\right)
\end{array}
\end{aligned}
$$

subject to

$$
\begin{array}{lr}
\sum_{j \in V^{\prime}} x_{i j} \leq 1 & \forall i \in D D \\
\sum_{i \in V^{\prime}} x_{i j} \leq 1 & \forall i \in A D \\
\sum_{i \in D D} \sum_{j \in V^{\prime}} x_{i j}=\sum_{i \in A D} \sum_{j \in V^{\prime}} x_{j i} & \\
\tau_{i}+t_{i j} x_{i j}+g^{i}\left(Y_{i}-y_{i}\right)-\left(l_{0}+g Q\right)\left(1-x_{i j}\right) \leq \tau_{j} \\
\quad \forall i \in F^{\prime}, \forall j \in V_{N+1}^{\prime}
\end{array}
$$

and (2)-(5), (7)-(14)

The objective function (15) minimizes the total recharging cost. The first term corresponds to the total cost of energy recharged along the route. The second is the total cost of initial charging at the depot. We assume that that all vehicles are recharged full using the cheapest (slowest) charger type at the depot overnight. The third is associated with the battery SoC at the end of the trip. The cost remaining energy on the battery is subtracted from the total spent since that energy is not consumed. Constraints (16) and (17) keep track of departures from the depots and arrival to the depots. Constraint (18) ensures that the number of departure depots used should be equal to the number of arrival depots used. Finally, constraints (19) ensure the time feasibility of arcs leaving the stations.

\section{EXPERIMENTAL STUDY}

We perform experimental tests using EVRPTW benchmark instances from the literature. We solve both Model 1 and Model 2 and compare the results. Our aim is to investigate the potential benefits of quick charging option and assess the additional complexity it brings in terms of solution time. The solutions are obtained by using IBM ILOG CPLEX v.12.6 optimization solver. All experiments are performed on an Intel Xeon E5 processor with $3.30 \mathrm{GHz}$ speed and $64 \mathrm{~GB}$ RAM, and 64-bit Windows 7 operating system.

\section{A. Experimental Design}

The EVRPTW data set consists of 36 small and 56 large instances generated by [16] based on VRPTW instances of [25]. We only use the small instances since the large problems are not tractable. The small instances include three subsets of 12 problems, each involving 5, 10, and 15 customers, and varying number of recharging stations. The customers are clustered $(C$ type), randomly distributed (R-type), and both clustered and randomly distributed (RC-type) over a 100×100 grid. Each set has also two subsets, type $1 \mathrm{xx}$ and type $2 \mathrm{xx}$, which differ by the length of the time windows and the vehicle load and battery capacities. The first four (five for RC group) characters of the problem ID show the problem type and the last four characters indicate the numbers of customers and stations, respectively.

The discharge rate $h$ is set to 1 . In the slow charge case (Model 1 ), the recharge rate and cost of unit energy is $g=c=1$. In Model 2 , we assume three types of chargers, namely slow, normal, and quick, and the charging rates and costs are $g=\{1 ; 0,18 ; 0,08\}$ and $c=\{1 ; 1,1 ; 1,2\}$, respectively. We limit the run time of CPLEX with 7200 seconds. If no optimal solution is obtained within this time limit, we report the upper bound.

\section{B. Numerical Results}

CPLEX was able to obtain the optimal solution of all instances in 5-customer set. The results are given in Table I. The columns '\#EV' and 'Cost' refer to the number of EVs and total energy cost, respectively, and 'Time' shows the run time in seconds.

TABLE I. RESULTS FOR 5-CUSTOMER INSTANCES

\begin{tabular}{|l|rrr|rrr|}
\hline & \multicolumn{3}{|c|}{ Single Charge } & \multicolumn{3}{c|}{ Quick Charge } \\
\cline { 2 - 7 } Problem & \#EV & Cost & Time & \#EV & Cost & Time \\
\hline C101C5-S3 & 2 & 257.75 & $<1$ & 2 & $\mathbf{2 5 0 . 6 9}$ & 94 \\
C103C5-S2 & 1 & 175.37 & $<1$ & 1 & 175.37 & 8 \\
C206C5-S4 & 1 & 242.56 & $<1$ & 1 & 242.56 & 1780 \\
C208C5-S3 & 1 & 164.34 & $<1$ & 1 & 164.34 & $<1$ \\
R104C5-S3 & 2 & 136.69 & $<1$ & 2 & 136.69 & 3 \\
R105C5-S3 & 2 & 156.08 & $<1$ & 2 & 156.08 & $<1$ \\
R202C5-S3 & 1 & 128.88 & $<1$ & 1 & 128.88 & 2 \\
R203C5-S4 & 1 & 179.06 & $<1$ & 1 & 179.06 & 4 \\
RC105C5-S4 & 2 & 233.77 & $<1$ & 2 & 233.77 & 1256 \\
RC108C5-S4 & 2 & 253.93 & $<1$ & 2 & 253.93 & 5 \\
RC204C5-S4 & 1 & 185.16 & $<1$ & 1 & 185.16 & 22 \\
RC208C5-S3 & 1 & 167.98 & $<1$ & 1 & 167.98 & 6 \\
\hline
\end{tabular}


We observe that quick charging option is beneficial only in problem C101C5 (highlighted in bold) and the optimal solution does not change in the remaining instances. Taking into consideration the small number of costumers and vehicles need, this result is not conclusive. On the other hand, we see that all problems in the single charge case are solved to optimality in less a second. When the quick charging is available the solution time may increase significantly, even if the problem size is very small.

TABLE II. RESULTS FOR 10-CUSTOMER INSTANCES

\begin{tabular}{|l|ccr|rrr|}
\hline & \multicolumn{3}{|c|}{ Single Charge } & \multicolumn{3}{c|}{ Quick Charge } \\
\cline { 2 - 7 } Problem & \#EV & Cost & Time & \#EV & Cost & Time \\
\hline C101C10-S5 & 3 & 388.25 & 44 & 3 & $\mathbf{3 8 2 . 9 3}$ & 222 \\
C104C10-S4 & 2 & 273.93 & 221 & $\mathbf{1}$ & $\mathbf{2 6 7 . 6 0}$ & 381 \\
C202C10-S5 & 1 & 304.06 & 2 & 1 & 304.06 & 427 \\
C205C10-S3 & 2 & 228.28 & $<1$ & $\mathbf{1}$ & 283.29 & 7200 \\
R102C10-S4 & 3 & 249.19 & $<1$ & 3 & 249.19 & 60 \\
R103C10-S3 & 2 & 206.30 & 62 & 2 & 206.30 & 7200 \\
R201C10-S4 & 1 & 241.51 & 232 & 1 & $\mathbf{2 4 1 . 2 5}$ & 7200 \\
R203C10-S5 & 1 & 222.64 & 18 & 1 & 222.64 & 5886 \\
RC102C10-S4 & 4 & 423.51 & $<1$ & 4 & $\mathbf{4 1 5 . 9 9}$ & 7200 \\
RC108C10-S4 & 3 & 347.90 & 3 & 3 & 347.90 & 351 \\
RC201C10-S4 & 1 & 412.86 & 2562 & 1 & 412.86 & 7200 \\
RC205C10-S4 & 2 & 325.98 & $<1$ & 2 & 325.98 & 58 \\
\hline
\end{tabular}

Table II reports the solutions for 10-customer instances. Cost values in bold indicate improvement in energy cost and \#EV values in bold and underlined show that one less vehicle is needed when quick charging is available. All the single charge problems are solved to optimality whereas in the quick charge case, CPLEX stopped when the time limit has been reached in 5 out of 12 instances. We observe that quick charging allows reduced energy cost in three instances and a saving of one vehicle in one instance. Furthermore, in one instance the solution improves in terms of both number of vehicles and energy cost. These results suggest that the logistics operations might benefit from quick charging option.

TABLE III. RESULTS FOR 15-CUSTOMER INSTANCES

\begin{tabular}{|l|ccr|ccc|}
\hline & \multicolumn{3}{|c|}{ Single Charge } & \multicolumn{3}{c|}{ Quick Charge } \\
\cline { 2 - 7 } Problem & \#EV & Cost & Time & \#EV & Cost & Time \\
\hline C103C15-S5 & 3 & 348.46 & 7200 & $\underline{\mathbf{2}}$ & 372.85 & 7200 \\
C106C15-S3 & 3 & 275.13 & 14 & $\underline{\mathbf{2}}$ & 312.77 & 7200 \\
C202C15-S5 & 2 & 383.62 & 298 & 2 & $\mathbf{3 8 1 . 2 3}$ & 7200 \\
C208C15-S4 & 2 & 300.55 & 69 & $\underline{\mathbf{1}}$ & 339.21 & 7200 \\
R102C15-S8 & 5 & 412.78 & 7200 & 5 & $\mathbf{4 1 2 . 2 5}$ & 7200 \\
R105C15-S6 & 4 & 336.15 & 51 & - & - & - \\
R202C15-S6 & 2 & 397.82 & 7200 & $\underline{\mathbf{1}}$ & 510.39 & 7200 \\
R209C15-S5 & 1 & 313.24 & 7200 & 1 & $\underline{323.43}$ & 7200 \\
RC103C15-S5 & 4 & 397.67 & 7200 & 4 & 397.67 & 7200 \\
RC108C15-S5 & 3 & 370.25 & 2105 & 3 & 370.25 & 7200 \\
RC202C15-S5 & 2 & 394.39 & 7200 & 2 & 394.39 & 7200 \\
RC204C15-S7 & 1 & 393.72 & 7200 & 1 & $\mathbf{3 9 3 . 0 2}$ & 7200 \\
\hline
\end{tabular}

Finally, the solutions for 15-customer instances are provided in Table III. We see that quick charging allows a reduction of one $\mathrm{EV}$ in the fleet in four instances whereas total energy cost can be slightly reduced in three instances. Note that CPLEX finds the optimal solution in five instances in the single charge case whereas optimality is not guaranteed in none of the 12 instances in the quick charge case. Furthermore, CPLEX is not able to find even a feasible solution for problem R105C15 in the latter case. More interestingly, for problem R209C15, the solution (best upper bound) provided by CPLEX for the quick charge case is worse than that of single charge case (shown in italic and underlined). This is surprising because a solution with single charge is always feasible for the quick charge case as well. These results reveal the increased complexity of Model 2 compared to Model 1 and also indicate the need for effective heuristic approaches for finding high quality solutions for realistic size problems.

\section{CONCLUSION}

In this study, we tackled The Electric Vehicle Routing Problem with Time Windows and formulated two mathematical models for different recharging options, namely single charge and quick charge cases. We attempted to solve small-size instances from the literature to present managerial insights about the potential benefits of quick charging and also to investigate the complexity of the problems. The results showed that quick charging might reduce the fleet size and decrease the cost of energy needed to operate the EVs.

Further research on this topic may focus on enhancing the mathematical models in order to improve the solution quality. However, the large problems will still be intractable. So, effective metaheuristic methods are needed to find near-optimal solutions fast. Another future research direction is to consider the heterogeneous fleet case. The heterogeneity within this context does not only arise from the vehicle capacities but from their batteries as well since the cruising range of EVs and discharge/recharge durations differ depending on their battery condition and age.

Furthermore, the battery performances also vary due to vehicle characteristics and environmental conditions, which may significant affect the routing decisions due to limited driving range of the EVs. In addition, we assume that recharging stations are always available with all charger types, which may not be true in real life and there may be queues in front of the chargers. So, variability in the recharging times arises as an interesting and challenging topic to be investigated within the stochastic context.

\section{REFERENCES}

[1] White Paper on Transport, 2011, "Roadmap to a single European transport area: Towards a competitive and resource-efficient transport system", Publications Office of the European Union, Luxembourg.

[2] US Environmental Protection Agency. "Sources of Greenhouse Gas Emissions". http://www.epa.gov/climatechange/ghgemissions/sources/ transportation.html 
[3] U.S. Department of Transportation, Bureau of Transportation Statistics, Freight Facts and Figures 2013, 2014.

[4] European Commission Mobility \& Transport. "Transport matters". http://ec.europa.eu/transport/strategies/facts-and-figures/allthemes/index_en.htm

[5] S. Edelstein, "Netherlands joins Norway in plans to end new gas, diesel car sales by 2025," 2016. http://www.greencarreports.com/news/ 1103507_netherlands-joins-norway-in-plans-to-end-new-gas-diesel-carsales-by-2025.

[6] S. Khan, "Germany pushes to ban petrol-fuelled cars within next 20 years,"2016, http://www.independent.co.uk/news/world/europe/germanypetrol-car-ban-no-combustion-diesel-vehicles-2030-a7354281.html.

[7] M. Yilmaz and P.T. Krein., "Review of battery charger topologies, charging power levels, and infrastructure for plug-in electric and hybrid vehicles", IEEE Transactions on Power Electronics, 28(5), pp. 2151-2169, 2013.

[8] A. Montoya, C. Guéret, J.E. Mendoza, amd J.G. Villegas, "The electric vehicle routing problem with nonlinear charging function," Transportation Research Part B: Methodological (in press).

[9] C.C. Chan, "The state of the art of electric, hybrid, and fuel cell vehicles," Proceedings of the IEEE, 95(4), 704-718, 2007.

[10] E. den Boer, S. Aarnink, F. Kleiner, and J. Pagenkopf, Zero emissions trucks: An overview of state-of-the-art technologies and their potential, Technical Deport, Delft, CE Delft, 2013.

[11] R.G. Conrad and M.A. Figliozzi, "The recharging vehicle routing problem," in Proceedings of the 2011 Industrial Engineering Research Conference, T. Doolen and E. Van Aken, Eds. 2011.

[12] H. Wang and R.L. Cheu, "Operations of a taxi fleet for advance reservations using electric vehicles and charging stations," Journal of the Transportation Research Board 2352, pp. 1-10, 2012.

[13] A. Omidvar and R. Tavakkoli-Moghaddam, "Sustainable vehicle routing: Strategies for congestion management and refueling scheduling," Proceedings of the IEEE International Energy Conference and Exhibition, Florence, pp. 1089-1094, 2012.

[14] O. Worley and D. Klabjan, "Simultaneous vehicle routing and charging station siting for commercial electric vehicles," Proceedings of the IEEE International Electric Vehicle Conference, Greenville, SC, pp. 1-3, 2012.

[15] S. Erdogan and E. Miller-Hooks, "A green vehicle routing problem," Transportation Research Part E, 48, pp. 100-114, 2012.

[16] M. Schneider, A. Stenger, and D. Goeke, "The electric vehicle routing problem with time windows and recharging stations," Transportation Science, 48, pp. 500-520, 2014.

[17] G. Desaulniers, F. Errico, S. Irnich, and M. Schneider, "Exact algorithms for electric vehicle-routing problems with time windows," Operations Research, 64(6), pp. 1388-1405, 2016.

[18] M. Keskin and B. Çatay, "Partial recharge strategies for the electric vehicle routing problem with time windows," Transportation Research Part C: Emerging Technologies, 65, pp. 111-127, 2016.

[19] Á.Felipe, M.T. Ortuño, G. Righini, and G. Tirado, "A heuristic approach for the green vehicle routing problem with multiple technologies and partial recharges," Transportation Research Part E: Logistics and Transportation Review, 71, pp. 111-128, 2014.

[20] M. Bruglieri, F. Pezzella, O. Pisacane, and S. Suraci, "A variable neighborhood search branching for the electric vehicle routing problem with time windows," Electronic Notes in Discrete Mathematics, 47, pp. 221-228, 2015.

[21] D. Goeke and M. Schneider, "Routing a mixed fleet of electric and conventional vehicles," European Journal of Operational Research, 245, pp. 81-99, 2015.

[22] G. Hiermann, J. Puchinger, S. Ropke, and R.F. Hartl, "The electric fleet size and mix vehicle routing problem with time windows and recharging stations," European Journal of Operational Research, 252, pp. 995-1018, 2016.
[23] S. Pelletier, O. Jabali, and G. Laporte, "50th anniversary invited articlegoods distribution with electric vehicles: review and research perspectives," Transportation Science, 50(1), 3-22, 2016.

[24] S. Pelletier, O. Jabali, and G. Laporte, "Battery electric vehicles for goods distribution: a survey of vehicle technology, market penetration, incentives and practices," unpublished.

[25] M.M. Solomon, "Algorithms for the vehicle routing and scheduling problems with time window constraints", Operations Research, 35(2), pp. 254-265, 1987. 